\title{
LAPLACE-BELTRAMI EIGENFUNCTION EXPANSION OF CORTICAL MANIFOLDS
}

\author{
Seongho Seo ${ }^{1} \quad$ Moo K. Chung $^{1,2,3, *}$ \\ ${ }^{1}$ Department of Brain and Cognitive Sciences, \\ Seoul National University, Korea \\ ${ }^{2}$ Department of Biostatistics and Medical Informatics, \\ ${ }^{3}$ Waisman Laboratory for Brain Imaging and Behavior, \\ University of Wisconsin, Madison, WI 53706, USA
}

\begin{abstract}
We represent a shape representation technique using the eigenfunctions of Laplace-Beltrami operator and compare the performance with the conventional spherical harmonic (SPHARM) representation. Cortical manifolds are represented as a linear combination of the Laplace-Beltrami eigenfunctions, which form orthonormal basis. Since the LaplaceBeltrami eigenfunctions reflect the intrinsic geometry of the manifolds, the new representation is supposed to more compactly represent the manifolds and outperform SPHARM representation. However, this is not demonstrated yet in brain imaging data. We demonstrate the superior reconstruction capability of the Laplace-Beltrami eigenfunction representation using cortical and amygdala surfaces as examples.
\end{abstract}

Index Terms - Amygdala, cortical surface, Fourier representation, Laplace-Beltrami eigenfunctions, spherical harmonics

\section{INTRODUCTION}

In medical image analysis, a shape representation is an important problem to understand morphological changes related to illness or disease. Anatomical surfaces are frequently realized as triangular meshes. Continuous parametrization of the surface coordinates helps computing surface measures such as curvature, local area elements or other geometric features which characterize shape variations.

Spherical harmonic (SPHARM) representation [1, 2, 3, $4,5,6]$ is probably the most widely used parametric surface model. Since the spherical harmonics can be easily computed, SPHARM has been widely used in a various neuroanatomical surfaces including ventricles [7], hippocampi [2, 4], and cortical surfaces [3, 6, 8].

However, the problem of SPHARM is that it requires to establish a smooth mapping from the surface to a unit sphere

The correspondence should be send to M.K.C. (mkchung@wisc.edu). This work was supported by the WCU Grant from the government of Korea to M.K.C. via surface flattening $[1,4,5,9,10]$. The spherical parameterization introduces metric distortion which compounds the reconstruction error.

On the other hand, the Laplace-Beltrami eigenfunction method does not require the spherical parameterization and possibly avoids metric distortion in the reconstruction process. We claim that the representation using the LaplaceBeltrami eigenfunctions has far less between-subject reconstruction error variability and converges faster to the ground truth with less number of basis than SPHARM.

\section{LAPLACE-BELTRAMI EXPANSION}

Consider a closed compact manifold $\mathcal{M} \subset \mathbb{R}^{3}$. Let $L^{2}(\mathcal{M})$ be the space of square integrable functions on $\mathcal{M}$ with the inner product

$$
\langle f, g\rangle_{\mathcal{M}}=\int_{\mathcal{M}} f(\mathbf{p}) g(\mathbf{p}) d \mu(\mathbf{p})
$$

where $\mu$ is the Lebesgue measure such that $\mu(\mathcal{M})$ is the total area of $\mathcal{M}$. The orthonormal basis in $L^{2}(\mathcal{M})$ is given by the eigenfunctions of

$$
\Delta_{\mathcal{M}} \psi_{j}=-\lambda \psi_{j},
$$

where $\Delta_{\mathcal{M}}$ is the Laplace-Beltrami operator in $\mathcal{M}[11,12]$. We order the eigenfunctions $\psi_{0}, \psi_{1}, \psi_{2}, \cdots$ according to the corresponding eigenvalues, $0=\lambda_{0}<\lambda_{1} \leq \lambda_{2} \leq \cdots$.

For surface coordinates $\mathbf{p}=\left(p^{1}, p^{2}, p^{3}\right)^{\prime}$, each coordinate function $p^{i}(\mathbf{p}) \in L^{2}(\mathcal{M})$ can be represented as a linear combination of the Laplace-Beltrami eigenfunctions:

$$
p^{i}(\mathbf{p})=\sum_{j=0}^{K-1} \beta_{j}^{i} \psi_{j}(\mathbf{p}),
$$

where $\beta_{j}^{i}=\left\langle p^{i}, \psi_{j}\right\rangle_{\mathcal{M}}$ are Fourier coefficients, and $K$ is the number of basis functions. We will refer to this representation as LB-expansion throughout this paper. 

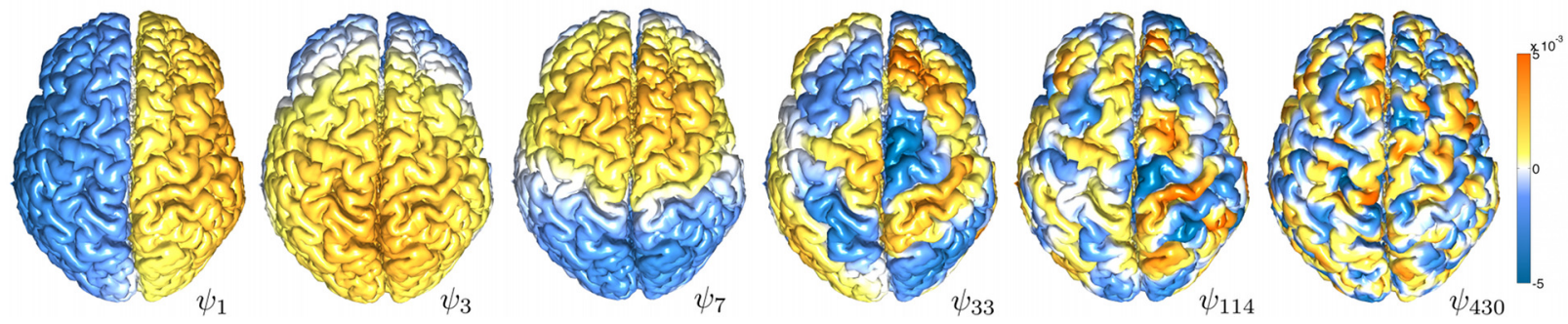

Fig. 1. Representative Laplace-Beltrami eigenfunctions for a cortical surface.

\section{SPHARM REPRESENTATION}

Let $S^{2}$ be a unit sphere. As a special case of the LaplaceBeltrami eigenfunctions, we have spherical harmonics $Y_{l m}$ of degree $l$ and order $m$ on the unit sphere $S^{2}$. There are $2 l+$ 1 eigenfunctions $Y_{l,-l}, \cdots, Y_{l, l}$ corresponding to the eigenvalue $\lambda_{l}=l(l+1)[13,14]$.

Consider parameterization $\mathbf{u} \in S^{2}$ :

$$
\mathbf{u}=\left(u^{1}, u^{2}, u^{3}\right)^{\prime}=(\sin \theta \cos \varphi, \sin \theta \sin \varphi, \cos \theta)^{\prime},
$$

where $(\theta, \varphi) \in \mathcal{N}=[0, \pi] \otimes[0,2 \pi)$. The polar angle $\theta$ is the angle from the north pole and the azimutal angle $\varphi$ is the angle along the horizontal cross-section. Assuming the manifold $\mathcal{M}$ is topologically equivalent to $S^{2}$, we can establish a smooth one-to-one mapping $\zeta: \mathbf{p} \in \mathcal{M} \rightarrow \mathbf{u} \in S^{2}$, and parameterize the surface coordinate $p^{i}$ with $(\theta, \varphi)$. Consequently, the surface coordinate can be expressed as

$$
p^{i}(\theta, \varphi)=\sum_{l=0}^{L} \sum_{m=-l}^{l} \beta_{l m}^{i} Y_{l m}(\theta, \varphi),
$$

where $\beta_{l m}^{i}=\left\langle p^{i}, Y_{l m}\right\rangle_{S^{2}}$ are Fourier coefficients.

\section{EXPERIMENTAL RESULTS}

\subsection{Numerical Implementation}

We have compared the LB-expansion with the SPHARM representation on amygdala and cortical surfaces. 3T-MRI data for 27 cortical surfaces and 41 amygdala surfaces were obtained from a 3-Tesla GE SIGNA scanner. The collected images went through several image processing, a detailed information of which can be found in [6] for cortical surfaces and in [10] for amydala surfaces. A deformable surface algorithm [9] was used to obtain the outer cortical surface by deforming from a spherical mesh with 40,962 vertices. The algorithm provides the same spherical parameterization for all cortical surfaces. Boundary of amygdala was extracted as a triangle mesh with approximately 2000-3000 vertices using a marching cubes algorithm. Then, the amygdala surface is mapped onto a sphere using a flattening algorithm proposed in [10].

The eigenfunctions of the Laplace-Beltrami operators are computed numerically using the Cotan formulation [12,
15]. The MATLAB code is freely available at http:// brainimaging.waisman.wisc. edu/ chung/lb.

The coefficients $\beta_{j}^{i}$ and $\beta_{l m}^{i}$ were estimated in the least squares fashion. For the LB-expansion, the eigenfunctions are computed for every surfaces, and representation for each subject is constructed with its own eigenfunctions. Fig. 1 shows the few representative eigenfunctions for a sample cortical surface. For the SPHARM, we simply obtain the basis from unit sphere by discretizing exact spherical harmonics with a given mesh parameterization.

For comparison, we matched the number of basis functions used in each representation: $K=(L+1)^{2}$. We used up to degree $L=80$ for the SPHARM which corresponds to $K=6561$ number of basis in the LB-expansion for cortical surfaces, and used up to degree $L=30$ and corresponding $K=961$ for amygdala surfaces.

\subsection{Comparison}

We compared both representations in terms of the mean and the variance of the reconstruction error for multiple subjects. The reconstruction error is defined as the average of the Euclidean distance between the original and the corresponding reconstructed surface coordinates:

$$
E=\frac{1}{n} \sum_{i=1}^{n}\left\|\mathbf{p}_{\mathbf{i}}-\widehat{\mathbf{p}}_{i}\right\|,
$$

where $n$ is the number of mesh vertices and $\widehat{\mathbf{p}}$ is the reconstructed surface. Fig. 2 shows the result of reconstruction for varying number of basis functions. For every subjects, then we computed the sample mean and the sample variance of $E$ as shown in Fig. 3, Table 1 and Table 2. The LB-expansion (red) shows smaller reconstruction errors than SPHARM (blue) at all degrees and converges faster. This implies that the LB-expansion requires smaller number of basis compared to SPHARM. The LB-expansion further shows less betweensubject variability compared to the SPHARM as shown in the size of error bars. These clearly demonstrates LB-expansion outperforms SPHARM in reconstruction accuracy.

\section{CONCLUSION}

We have presented LB-expansion for representing cortical manifolds. The representation is analytically constructed 


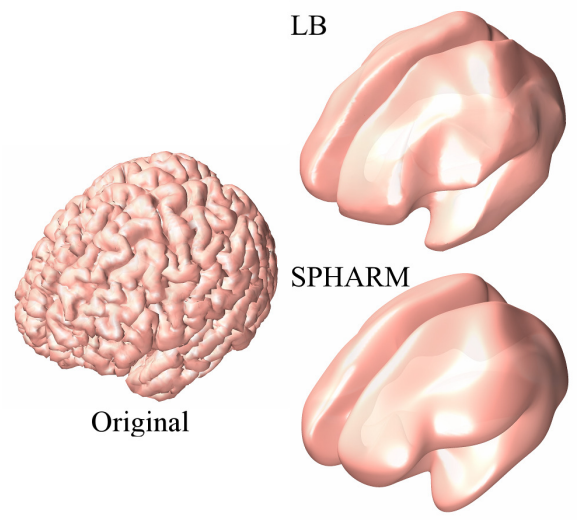

$10 \quad 2.9002$

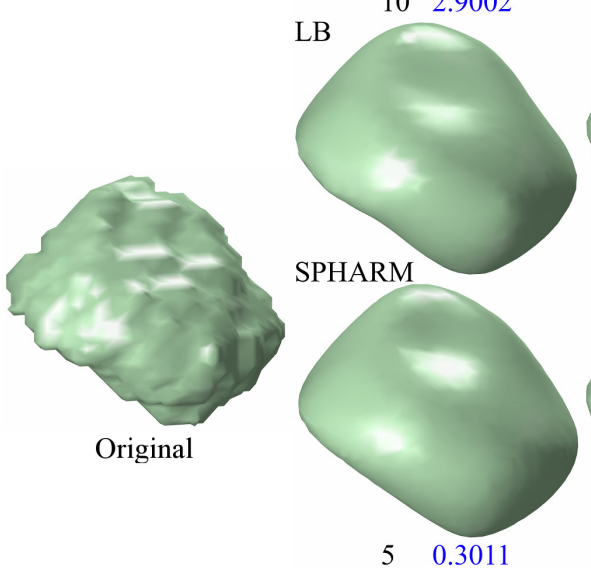

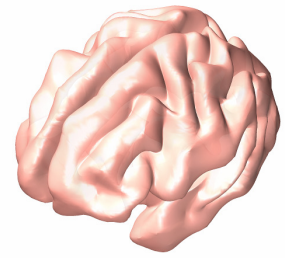
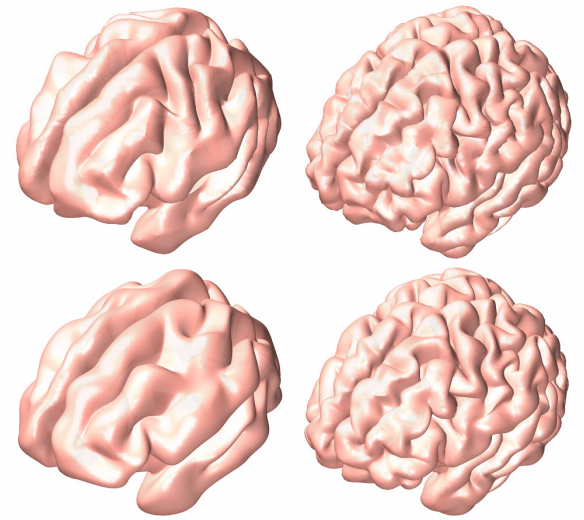

$40 \quad 1.4004$
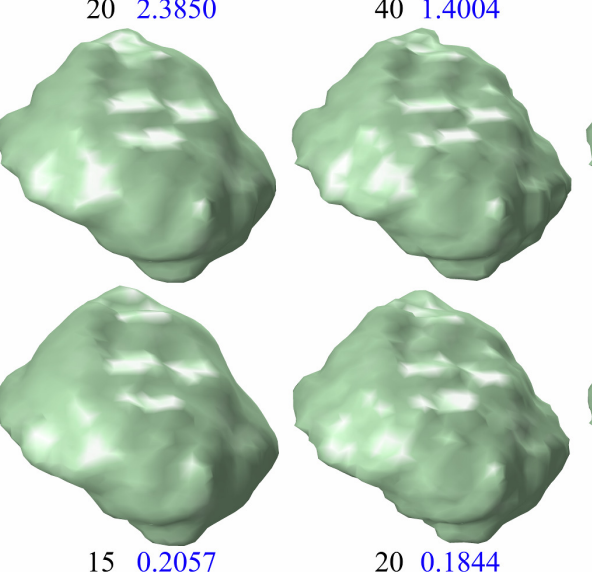

200.1844
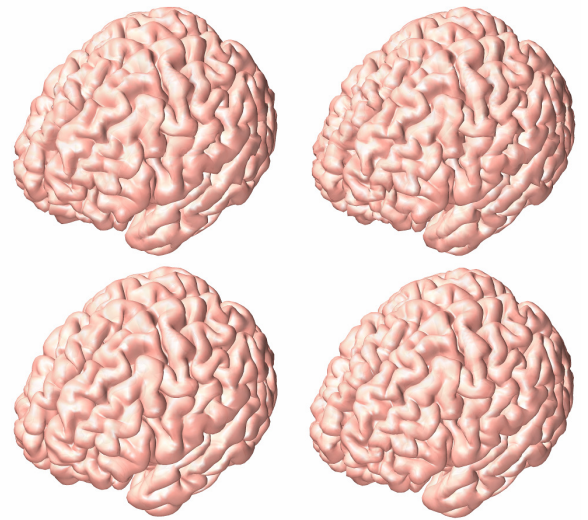

600.9342

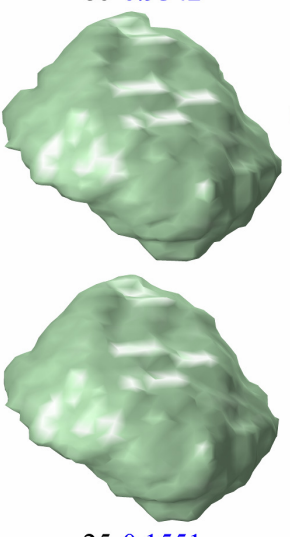

250.1551
800.6976
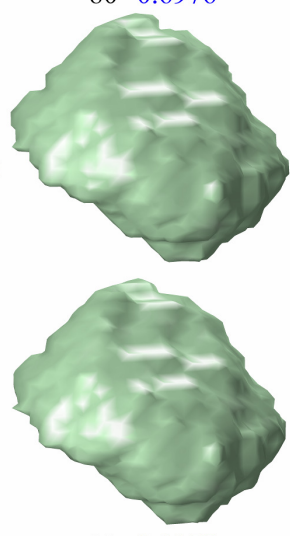

$30 \quad 0.0978$

Fig. 2. Comparison of LB-expansion and SPHARM representation of cortical surface (1st and 2nd rows) and amygdala (3rd and 4th rows) with increasing degree $l$ (Black colored numbers), which corresponds to $K=(L+1)^{2}$ number of basis. Blue colored numbers are the average Euclidean distance between two representations in $\mathrm{mm}$.

Table 1. Average Euclidean Distance (Cortical surface)

\begin{tabular}{|c|c|c|c|}
\hline$K$ & $L$ & LB & SPHARM \\
\hline 121 & 10 & $5.3821 \pm 0.0575$ & $6.3234 \pm 0.1628$ \\
441 & 20 & $3.1113 \pm 0.0062$ & $4.0239 \pm 0.1161$ \\
961 & 30 & $2.0498 \pm 0.0006$ & $2.7677 \pm 0.1175$ \\
1681 & 40 & $1.5138 \pm 0.0149$ & $2.0877 \pm 0.0962$ \\
2601 & 50 & $1.2185 \pm 0.0294$ & $1.6722 \pm 0.0877$ \\
3721 & 60 & $1.0284 \pm 0.0311$ & $1.3971 \pm 0.0821$ \\
5041 & 70 & $0.8953 \pm 0.0300$ & $1.2068 \pm 0.0743$ \\
6561 & 80 & $0.7992 \pm 0.0264$ & $1.0686 \pm 0.0685$ \\
\hline
\end{tabular}

using Lapalce-Beltrami eigenfunctions avoiding spherical parameterization. This drastically improves the reconstruction errors and make LB-expansion converges faster than SPHARM. Application to cortical surfaces and amygdala shows that the LB-expansion outperforms the traditional SPHARM representation in terms of reconstruction errors.
Table 2. Average Euclidean Distance (Amygdala surface)

\begin{tabular}{|c|c|c|c|}
\hline$K$ & $L$ & LB & SPHARM \\
\hline 36 & 5 & $0.2925 \pm 0.0293$ & $0.4261 \pm 0.0432$ \\
121 & 10 & $0.1781 \pm 0.0191$ & $0.3012 \pm 0.0305$ \\
256 & 15 & $0.1311 \pm 0.0103$ & $0.2491 \pm 0.0217$ \\
441 & 20 & $0.0918 \pm 0.0073$ & $0.2065 \pm 0.0165$ \\
676 & 25 & $0.0556 \pm 0.0070$ & $0.1609 \pm 0.0149$ \\
961 & 30 & $0.0257 \pm 0.0064$ & $0.1004 \pm 0.0184$ \\
\hline
\end{tabular}

\section{REFERENCES}

[1] C. Brechbühler, G. Gerig, and O. Kubler, "Parametrization of closed surfaces for 3d shape description," Computer Vision and Image Understanding, vol. 61, pp. 154-170, 1995.

[2] A. Kelemen, G. Szekely, and G. Gerig, "Elastic modelbased segmentation of 3-d neuroradiological data sets," 

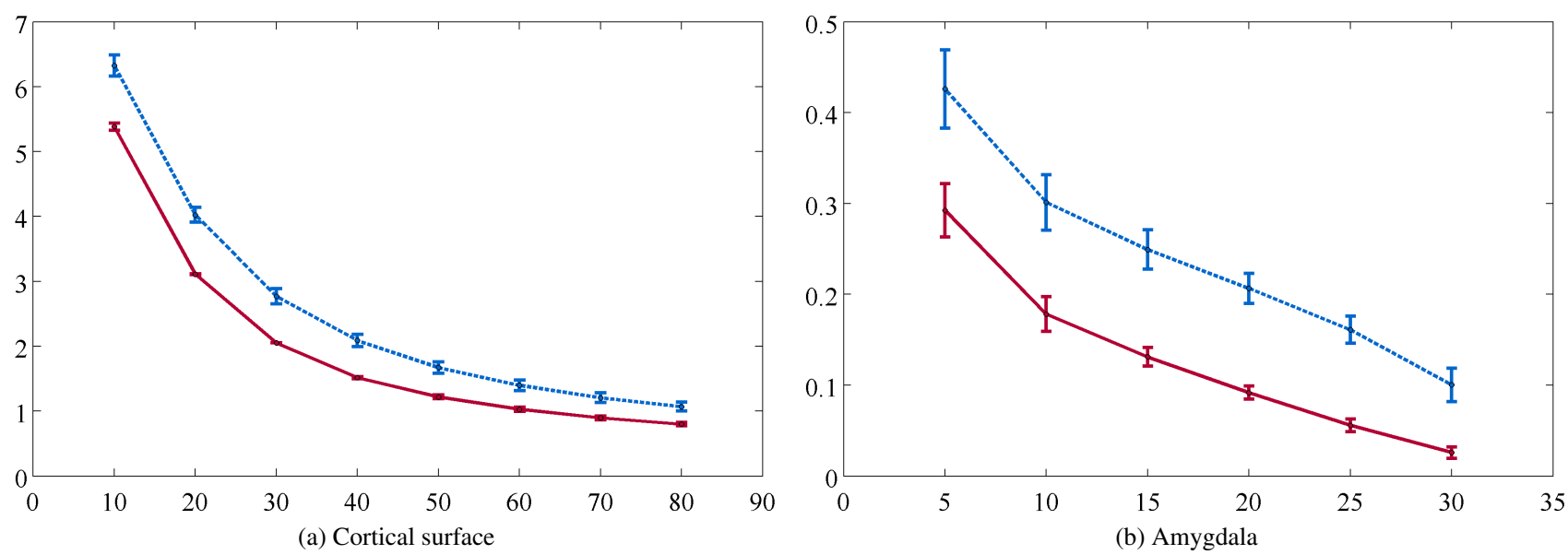

Fig. 3. Comparison of reconstruction accuracy over varying degree $L$ and corresponding $K=(L+1)^{2}$ number of basis. The accuracy is measured in terms of average Euclidean distance between corresponding mesh vertices. Red shows the LaplaceBeltrami (LB) expansion with $K$ number of basis, and blue SPHARM representation of degree $L$. Each node and bar indicates the mean and the standard deviation of average Euclidean distance for the population of surfaces.

IEEE Transactions on Medical Imaging, vol. 18, pp. 828-839, 1999.

[3] X. Gu, Y.L. Wang, T.F. Chan, T.M. Thompson, and S.T. Yau, "Genus zero surface conformal mapping and its application to brain surface mapping," IEEE Transactions on Medical Imaging, vol. 23, pp. 1-10, 2004.

[4] L. Shen, J. Ford, F. Makedon, and A. Saykin, "surfacebased approach for classification of $3 \mathrm{~d}$ neuroanatomical structures," Intelligent Data Analysis, vol. 8, pp. 519$542,2004$.

[5] M. Styner, I. Oguz, S. Xu, C. Brechbuhler, D. Pantazis, J. Levitt, M. Shenton, and G. Gerig, "Framework for the statistical shape analysis of brain structures using spharm-pdm," in Insight Journal, Special Edition on the Open Science Workshop at MICCAI, 2006, http://hdl.handle.net/1926/215.

[6] M. K. Chung, K. M. Dalton, L. Shen, A. C. Evans, and R. J. Davidson, "Weighted Fourier representation and its application to quantifying the amount of gray matter," IEEE Transactions on Medical Imaging, vol. 26, pp. 566-581, 2007.

[7] G. Gerig, M. Styner, D. Jones, D. Weinberger, and J. Lieberman, "Shape analysis of brain ventricles using spharm," in MMBIA, 2001, pp. 171-178.

[8] R. A. Yotter, P. M. Thompson, I. Nenadic, and C. Gaser, "Estimating local surface complexity maps using spherical harmonic reconstructions," in Medical Image Computing and Computer-Assisted Intervention - MICCAI
2010. 2010, vol. 6362 of Lecture Notes in Computer Science, pp. 169-176, Springer.

[9] J.D. MacDonald, N. Kabani, D. Avis, and A.C. Evans, "Automated 3-D extraction of inner and outer surfaces of cerebral cortex from mri," NeuroImage, vol. 12, pp. 340-356, 2000.

[10] M. K. Chung, A. Qiu, B. M. Nacewicz, K. M. Dalton, S. Pollak, and R. J. Davidson, "Tiling manifolds with orthonormal basis.," in 2nd MICCAI Workshop on Mathematical Foundations of Computational Anatomy (MFCA)., 2008.

[11] B. Lévy, "Laplace-Beltrami Eigenfunctions: Towards an Algorithm that Understands Geometry," in IEEE International Conference on Shape Modeling and Applications. 2006, p. 13, IEEE.

[12] S. Seo, M. K. Chung, and H. K. Vorperian, "Heat kernel smoothing using laplace-beltrami eigenfunctions," in Medical Image Computing and Computer-Assisted Intervention - MICCAI 2010. 2010, vol. 6363 of Lecture Notes in Computer Science, pp. 505-512, Springer.

[13] R. Courant and D. Hilbert., Methods of Mathematical Physics, Volume I., Interscience, New York, 1953.

[14] G. Wahba, Spline models for observational data, SIAM, 1990.

[15] A. Qiu, D. Bitouk, and M. I. Miller, "Smooth functional and structural maps on the neocortex via orthonormal bases of the Laplace-Beltrami operator," IEEE Transactions on Medical Imaging, vol. 25, pp. 1296-1306, 2006. 